UDC 51:621.891

DOI: $10.32326 / 1814-9146-2019-81-4-501-512$

\title{
A MATHEMATICAL MODEL OF NONSTATIONARY MOTION OF A VISCOELASTIC FLUID IN ROLLER BEARINGS
}

\author{
(C) 2019 Zhurba Eremeeva I.A., Scerrato D., Cardillo C., Tran A. \\ International Research Center for the Mathematics and Mechanics \\ of Complex Systems Universita degli Studi dell'Aquila, L'Aquila, Italia
}

eremeyeva.inna@gmail.com

Received by the Editor 23.09.2019

\begin{abstract}
Nowadays, the emergence of new lubricants requires an enhancement of the rheological models and methods used for solution of corresponding initial boundaryvalue problems. In particular, models that take into account viscoelastic properties are of great interest. In the present paper we consider the mathematical model of nonstationary motion of a viscoelastic fluid in roller bearings. We used the Maxwell fluid model for the modeling of fluid properties. The viscoelastic properties are exhibited by many lubricants that use polymer additives. In addition, viscoelastic properties can be essential at high fluid speeds. Also, viscoelastic properties can be significant in the case of thin gaps. Maxwell's model is one of the most common models of viscoelastic materials. It combines the relative simplicity of constitutive equations with the ability to describe a stress relaxation. In addition, viscoelastic fluids also allow us to describe some effects that are missing in the case of viscous fluid. An example it is worth to mention the Weissenberg effect and a number of others. In particular, such effects can be used to increase the efficiency of the film carrier in the sliding bearings. Here we introduced characteristic assumptions on the form of the flow, allowing to significantly simplify the solution of the problem. We consider so-called self-similar solutions, which allows us to get a solution in an analytical form. As a result these assumptions, the formulae for pressure and friction forces are derived. Their dependency on time and Deborah number is analyzed. The limiting values of the flow characteristics were obtained. The latter can be used for steady state of the flow regime. Differences from the case of Newtonian fluid are discussed. It is shown that viscoelastic properties are most evident at the initial stage of flow, when the effects of non-stationarity are most important.
\end{abstract}

Keywords: viscoelastic fluid, roller bearing, friction, self-similar solution.

\section{Introduction}

In theoretical studies of lubrication, it is often assumed that a lubricant behaves as a true (Newtonian) fluid even at very high shear rates, which are characteristic of bearings, and that various stress components resulting in the fluid from the displacement of its boundaries can be determined based on classical hydrodynamics. However, many of the modern lubricants possess noticeable elasticity in shear, and, in laminar flow, these materials demonstrate some new phenomena (for example, "the Weissenberg effect" or "the normal 
pressure phenomenon"), which cannot be explained in the framework of the classical theory. For a flow of a particular geometrical configuration, the "normal pressure phenomenon" can be used for forming a carrying film in roller bearings.

The topic of stationary motion of viscoelastic lubricants is fairly well studied (see [1-3]. In the case of stationary shear flow similar to flows in most bearings with liquid lubricants, non-Newtonian fluids differ very little from Newtonian ones. For stationary shear flows, normal stresses are too small to give any tangible contribution into the carrying capacity of the layer in the conditions of the geometry of such bearings. Lubricants have been studied in numerous works (e.g., [4-15]), taking account of their viscoelastic properties. The hydrodynamics of viscoelastic fluids was considered in [16-19]. The approach used here was also applied in [20].

It appears that the behavior of viscoelastic lubricants can differ substantially from that of Newtonian lubricants only in nonstationary conditions. That is why solution of the problem of nonstationary flow of viscoelastic lubricants in roller bearings is of both theoretical and practical importance.

\section{Basic assumptions}

In the present paper, the problem of a nonstationary flow of a viscoelastic lubricant between a slider and a bearing pilot (fig. 1) is analyzed. The slider is assumed to remain static, whereas the pilot moves at a velocity of $\widetilde{u}^{0}+u^{*}\left(t^{\prime}\right)$, where $\widetilde{u}^{0}=$ const, $u^{*}\left(t^{\prime}\right)$ is perturbation velocity of the of the pilot.

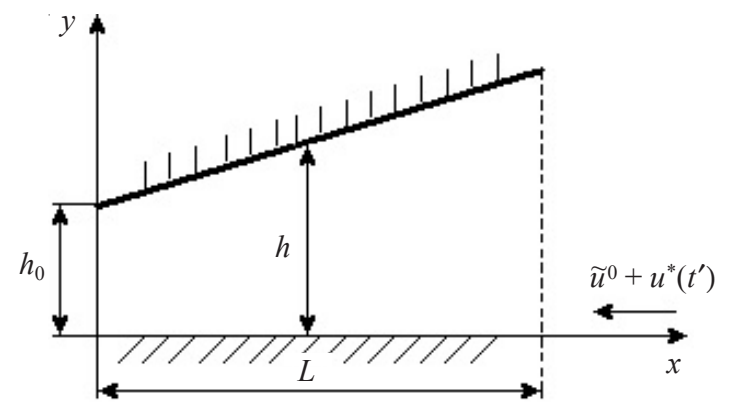

Fig.1. Configuration of the bearing

To find an analytical solution of the problem connected with stationary motion of the pilot, a number of conventional assumptions and simplifications are made:

1. Pressure is nearly constant through the film thickness, as defined by equation $h^{\prime}=h_{0}+$ $+x^{\prime} \operatorname{tg} \alpha$.

2. The lubricant film thickness is small as compared with its length, thus, according to Reynolds, it can be assumed that velocity $V_{y^{\prime}}^{\prime}$ of the fluid and in the direction normal to the surface is small enough as compared with velocity $V_{x^{\prime}}^{\prime}$ along the surfaces. Moreover, the variation of velocity $V_{x^{\prime}}^{\prime}$ in direction $x^{\prime}$ is negligible as compared with that in direction $y^{\prime}$. 3. The characteristics of a Maxwellian fluid can be described using the following equation $[2,3]$ :

$$
\frac{\partial V_{x^{\prime}}^{\prime}}{\partial y^{\prime}}=\frac{\tau^{\prime}}{\mu}+\frac{1}{G} \cdot \frac{\partial \tau^{\prime}}{\partial t^{\prime}},
$$

where $G$ is elastic modulus, $\mu$ is viscosity, $\mu / G=\lambda$ is relaxation time of the fluid, $\tau^{\prime}$ is tangential stress. 


\section{Basic equations and boundary conditions}

With the above assumptions, the analysis of equilibrium of a fluid element situated between the surfaces of the bearing results in the following equation:

$$
\frac{\partial \tau^{\prime}}{\partial y^{\prime}}=\frac{\partial p^{\prime}}{\partial x^{\prime}}+\rho \frac{\partial V_{x^{\prime}}^{\prime}}{\partial t^{\prime}}
$$

where $p^{\prime}$ is hydrodynamic pressure and $\rho$ is density.

In analyzing the system in question, equations (1) and (2) are complemented with a continuity equation:

$$
\frac{\partial V_{y^{\prime}}^{\prime}}{\partial y^{\prime}}+\frac{\partial V_{x^{\prime}}^{\prime}}{\partial x^{\prime}}=0
$$

The solution of the analyzed problem will be sought in the form of a sum of two solutions:

$$
V_{x^{\prime}}^{\prime}=V_{x^{\prime}}+V_{x^{\prime}}^{0}, \quad V_{y^{\prime}}^{\prime}=V_{y^{\prime}}+V_{y^{\prime}}^{0}, \quad p^{\prime}=p_{\mathrm{ns}}^{\prime}+p_{0}^{\prime}, \quad \tau^{\prime}=\tau_{\mathrm{ns}}^{\prime}+\tau_{0}^{\prime},
$$

where $V_{x^{\prime}}^{0}, V_{y^{\prime}}^{0}, p_{0}^{\prime}, \tau_{0}^{\prime}$ is solution of a stationary problem corresponding to the motion of the bearing pilot at a constant velocity $\widetilde{u}^{0}$, and $V_{y^{\prime}}, V_{x^{\prime}}, p_{\mathrm{ns}}^{\prime}, \tau_{\mathrm{ns}}^{\prime}$ is a solution of a nonstationary problem corresponding to the motion of the pilot at a velocity $u^{*}\left(t^{\prime}\right)$.

Substituting $\widetilde{u}^{0} \partial / \partial x^{\prime}$ for $\partial / \partial t^{\prime}$ in equation (1) of the stationary problem, to determine functions $V_{x^{\prime}}^{0}, V_{y^{\prime}}^{0}, p_{0}^{\prime}, \tau_{0}^{\prime}$ with the account of (1), (2) and (3), the following equation system is obtained:

$$
\frac{\partial V_{x^{\prime}}^{0}}{\partial y^{\prime}}=\frac{\tau_{0}^{\prime}}{\mu}+\frac{\tilde{u}^{0}}{G} \cdot \frac{\partial \tau_{0}^{\prime}}{\partial x^{\prime}}, \quad \frac{\partial V_{y^{\prime}}^{0}}{\partial y^{\prime}}+\frac{\partial V_{x^{\prime}}^{0}}{\partial x^{\prime}}=0, \quad \frac{\partial \tau_{0}^{\prime}}{\partial y^{\prime}}=\frac{d p_{0}^{\prime}}{\partial x^{\prime}},
$$

and to determine functions $V_{y^{\prime}}, V_{x^{\prime}}, p_{\mathrm{ns}}^{\prime}, \tau_{\mathrm{ns}}^{\prime}$, in equations (1), (2) and (3) $V_{y^{\prime}}, V_{x^{\prime}}, p_{\mathrm{ns}}^{\prime}, \tau_{\mathrm{ns}}^{\prime}$ will be substituted for $V_{y^{\prime}}^{\prime}, V_{x^{\prime}}^{\prime}, p^{\prime}, \tau^{\prime}$, respectively.

Equation system (5) is solved with the following boundary conditions:

$$
\begin{gathered}
V_{y^{\prime}}^{0}=0, \quad V_{x^{\prime}}^{0}=-\widetilde{u}^{0} \text { for } y^{\prime}=0, \\
V_{y^{\prime}}^{0}=0, \quad V_{x^{\prime}}^{0}=0 \text { for } y^{\prime}=h^{\prime}\left(x^{\prime}\right), \\
p_{0}^{\prime}=P_{A} \text { for } x^{\prime}=0, x^{\prime}=l .
\end{gathered}
$$

It is assumed that a lubricant arrives at the bearing in the case of total relaxation of elastic strain. If this assumption is applied to the entire fluid at the moment it gets into the bearing, boundary conditions (6) will be supplemented with the following conditions:

$$
\frac{d^{2} p_{0}^{\prime}}{d x^{\prime 2}}=0, \quad \frac{d c_{0}}{d x}=0 \text { for } x=0 .
$$

Now dimensionless variables will be considered according to the following formulas:

$$
\begin{gathered}
x^{\prime}=l x, \quad y^{\prime}=h_{0} y, \quad V_{x^{\prime}}^{0}=\widetilde{u}^{0} v^{0}, \quad V_{y^{\prime}}^{0}=\widetilde{u}^{0} \varepsilon u^{0}, \quad h^{\prime}\left(x^{\prime}\right)=h_{0} h(x), \quad \varepsilon=\frac{h_{0}}{l}, \\
p_{0}^{\prime}=p^{*} p_{0}, \quad p^{*}=\frac{\mu \widetilde{u} l}{h_{0}^{2}},
\end{gathered}
$$

where $l$ is length of the bearing pilot.

Then equation system (5) and boundary conditions (6), (7) can be written as: 


$$
\begin{gathered}
\frac{\partial^{2} v^{0}}{\partial y^{2}}=\frac{\partial p_{0}}{\partial x}+\beta \frac{\partial^{2} p_{0}}{\partial x^{2}}, \quad \frac{\partial u^{0}}{\partial y}+\frac{\partial v^{0}}{\partial x}=0, \quad \beta=\frac{\mu \tilde{u}^{0}}{G h_{0}}, \\
v^{0}=0, \quad u^{0}=0 \text { for } y=h(x), \quad v^{0}=-1, \quad u^{0}=0 \text { for } y=0, \\
p_{0}=\frac{P_{A}}{p^{*}} \text { for } x=0, x=1, \quad \frac{d^{2} p_{0}}{d x^{2}}=0 \text { for } x=0 .
\end{gathered}
$$

\section{Constructing a self-similar solution}

An exact self-similar solution of problem (9), (10) is sought in the following form [2]:

$$
\begin{gathered}
v^{0}=\frac{\partial \psi^{0}}{\partial y}+V^{0}(x, y), \quad u^{0}=-\frac{\partial \psi^{0}}{\partial x}+U^{0}(x, y), \\
\psi^{0}=\widetilde{\psi}^{0}(\xi), \quad U^{0}=\widetilde{u}_{0}(\xi) h^{\prime}(x), \quad V^{0}=\widetilde{v}_{0}(\xi), \\
\xi=\frac{y}{h(x)}, \quad \frac{d p_{0}}{d x}+\beta \frac{d^{2} p_{0}}{d x^{2}}=\frac{\widetilde{c}_{1}^{0}}{h^{2}}+\frac{\widetilde{c}_{2}^{0}}{h^{3}} .
\end{gathered}
$$

Substitution of (11) into (9), (10) yields the following system of ordinary differential equations and the related boundary conditions:

$$
\begin{gathered}
\widetilde{\psi}^{0^{\prime \prime \prime}}=\widetilde{c}_{2}^{0}, \quad \widetilde{v}^{0^{\prime \prime}}=\widetilde{c}_{1}^{0}, \quad u_{0}^{\prime}-\xi \widetilde{v}_{0}^{\prime}=0, \\
\widetilde{\psi}^{0^{\prime}}=0, \quad \widetilde{u}_{0}=0, \quad \widetilde{v}_{0}=0 \text { for } \xi=1, \quad \widetilde{\psi}^{0^{\prime}}=0, \quad \widetilde{u}_{0}=0, \quad \widetilde{v}_{0}=-1 \text { for } \xi=0, \\
p_{0}=\frac{P_{A}}{p^{*}} \text { for } x=0, x=1, \quad \frac{d^{2} p_{0}}{d x^{2}}=0 \text { for } x=0, \int_{0}^{1} \widetilde{v}_{0}(\xi) d \xi=0 .
\end{gathered}
$$

A solution of problem (12), (13) is found by direct integration with the following result:

$$
\widetilde{\psi}^{0^{\prime}}=\widetilde{c}_{2}^{0} \frac{\xi^{2}}{2}+c_{1}^{0} \xi+c_{2}^{0}, \quad \widetilde{v}_{0}=c_{1}^{0} \frac{\xi^{2}}{2}+c_{3}^{0} \xi+c_{4}^{0}, \quad \tilde{u}_{0}=\int_{0}^{\xi} \xi \widetilde{v}_{0}^{\prime}(\xi) d \xi,
$$

where $\widetilde{c}_{1}^{0}=6, c_{2}^{0}=0, c_{3}^{0}=-4, c_{1}^{0}=-\widetilde{c}_{2}^{0} / 2, c_{4}^{0}=-1$,

$$
\widetilde{c}_{2}^{0}=\frac{1}{J_{3}(1)}\left[\beta \int_{0}^{1} \frac{d^{2} p_{0}}{d x^{2}} d x-6 J_{2}(1)\right], \quad J_{k}(x)=\int_{0}^{x} \frac{d x}{h^{k}(x)} .
$$

For hydrodynamic pressure with the account of the boundary conditions for $p_{0}$, the following final expression is obtained:

$$
p_{0}=\frac{6\left((-2 \exp (-x / \beta)+2 x \exp (-1 / \beta)-2 x+2) \beta^{2}+x^{2}-x\right) \eta}{(-6 \eta+6 \eta \exp (-1 / \beta)) \beta^{2}+6 \beta \eta-3 \eta+2}+\frac{P_{A}}{p^{*}} .
$$

Knowing $p_{0}$, pressure force $N_{0}$ for the case of stationary motion can be found:

$$
\begin{gathered}
N_{0}=\frac{\mu \widetilde{u}^{0} l^{2}}{h_{0}^{2}} \int_{0}^{1} p_{0} d x=\frac{\mu \widetilde{u}^{0} l^{2}}{h_{0}^{2}} \times \\
\times \frac{-12 \beta^{3} \eta+12 \beta^{3} \eta \exp (1 / \beta)-6 \beta^{2} \eta \exp (1 / \beta)-6 \beta^{2} \eta+\eta \exp (1 / \beta)}{3 \eta \exp (1 / \beta)-6 \beta^{2} \eta-2 \exp (1 / \beta)-6 \beta \eta \exp (1 / \beta)+6 \beta^{2} \eta \exp (1 / \beta)}+P_{A} .
\end{gathered}
$$


For the case of stationary motion, the friction force is defined by the following expression:

$$
F_{\mathrm{fr}}^{0}=\left.\int_{0}^{l} \tau_{0}^{\prime}\right|_{y^{\prime}=0} d x^{\prime}=\int_{0}^{l} c_{0}^{\prime}\left(x^{\prime}\right) d x^{\prime}=\frac{\mu \tilde{u}^{0} l}{h_{0}} \int_{0}^{l} c_{0}(x) d x,
$$

where constant $c_{0}^{\prime}=c_{0} \widetilde{u}^{0} \mu / h_{0}$ results from integrating the third equation of system (5), and $c_{0}$ is determined from the following expression:

$$
\frac{c_{1}^{0}}{h^{2}}+\frac{c_{3}^{0}}{h}=c_{0}(x)+\beta \frac{d c_{0}}{d x} \text {. }
$$

Solving the resulting equation with boundary condition $d c_{0} / d x=0$ for $x=0$, one obtains:

$$
c_{0}=-2 \frac{-6 \beta^{2} \eta+4 \eta \beta+2 \eta x-3 \eta+1+6 \exp (-1 / \beta) \eta \beta^{2}+2 \eta \beta \exp (-x / \beta)}{-6 \beta^{2} \eta+6 \exp (-1 / \beta) \eta \beta^{2}+6 \eta \beta+2-3 \eta} .
$$

The final expression for determining the friction force with the account of (15) will be:

$$
\begin{gathered}
F_{\mathrm{fr}}^{0}=\frac{\mu \tilde{u}^{0} l}{h_{0}} \times \\
\times\left[-2 \frac{-4 \beta^{2} \eta-4 \eta \beta \exp (1 / \beta)+2 \eta \exp (1 / \beta)+4 \exp (1 / \beta) \eta \beta^{2}-\exp (1 / \beta)}{-6 \beta^{2} \eta+3 \eta \exp (1 / \beta)-2 \exp (1 / \beta)-6 \eta \beta \exp (1 / \beta)+6 \eta \beta^{2} \exp (1 / \beta)}\right] .
\end{gathered}
$$

Now the nonstationary problem will be analyzed.

The nonlinear term in the right-hand side of equation (2) is averaged along the thickness of the lubricant layer using the Slezkin-Targ method. The following designation is introduced:

$$
A^{\prime}=\frac{1}{h^{\prime}\left(x^{\prime}\right)} \int_{0}^{h^{\prime}\left(x^{\prime}\right)} \rho \frac{\partial V_{x^{\prime}}}{\partial t^{\prime}} d y^{\prime}
$$

Thus, after integrating equation (2) for the case of nonstationary motion with the account of (3), the following expression is obtained:

$$
\tau_{\mathrm{ns}}^{\prime}=\frac{\partial p_{\mathrm{ns}}^{\prime}}{\partial x^{\prime}} y^{\prime}+A^{\prime}\left(x^{\prime}, t^{\prime}\right) y^{\prime}+c^{\prime}\left(x^{\prime}, t^{\prime}\right)
$$

For the case of a Maxwellian fluid, where its flow characteristics are defined by equation (1), the velocity gradient will be expressed as:

$$
\frac{\partial V_{x^{\prime}}}{\partial y^{\prime}}=\frac{1}{\mu}\left(\frac{\partial p_{\mathrm{ns}}^{\prime}}{\partial x^{\prime}} y^{\prime}+A^{\prime}\left(x^{\prime}, t^{\prime}\right) y^{\prime}+c^{\prime}\left(x^{\prime}, t^{\prime}\right)\right)+\frac{1}{G}\left(\frac{\partial^{2} p_{\mathrm{ns}}^{\prime}}{\partial x^{\prime} \partial t^{\prime}} y^{\prime}+\frac{\partial A^{\prime}}{\partial t^{\prime}} y^{\prime}+\frac{\partial c^{\prime}}{\partial t^{\prime}}\right) .
$$

Differentiating both sides of equation (19) for $y^{\prime}$, one obtains:

$$
\frac{\partial^{2} V_{x^{\prime}}}{\partial y^{\prime 2}}=\frac{1}{\mu}\left(\frac{\partial p_{\mathrm{ns}}^{\prime}}{\partial x^{\prime}}+A^{\prime}\left(x^{\prime}, t^{\prime}\right)\right)+\frac{1}{G}\left(\frac{\partial^{2} p_{\mathrm{ns}}^{\prime}}{\partial x^{\prime} \partial t^{\prime}}+\frac{\partial A^{\prime}}{\partial t^{\prime}}\right) .
$$

Let us consider now the dimensionless variables according to the formulas analogous to (7):

$$
\begin{gathered}
x^{\prime}=l x, \quad y^{\prime}=h_{0} y, \quad V_{x^{\prime}}=\widetilde{u}^{0} v, \quad V_{y^{\prime}}=\widetilde{u}^{0} \varepsilon u, \quad h^{\prime}\left(x^{\prime}\right)=h_{0} h(x), \quad \varepsilon=\frac{h_{0}}{l}, \\
p^{\prime}=P \cdot p^{*}, \quad p^{*}=\frac{\mu \widetilde{u}^{0} l}{h_{0}^{2}}, \quad t=t \cdot t^{*}, \quad t^{*}=\frac{h_{0}}{\widetilde{u}^{0}}, \quad c^{\prime}=c^{*} \cdot c, \quad c^{*}=\frac{\widetilde{u}^{0} \mu}{h_{0}} .
\end{gathered}
$$


For the nonstationary problem, with the account of (21), the following equation system and the related boundary conditions are obtained:

$$
\begin{gathered}
\frac{\partial^{2} v}{\partial y^{2}}=\frac{\partial P}{\partial x}+\operatorname{Re} \cdot A+\beta \frac{\partial^{2} P}{\partial x \partial t}+\operatorname{Re} \cdot \beta \cdot \frac{\partial A}{\partial t} \\
\frac{\partial u}{\partial y}+\frac{\partial v}{\partial x}=0, \quad \operatorname{Re}=\frac{\rho \tilde{u}^{0} h_{0}}{\mu}, \quad \beta=\frac{\mu \tilde{u}^{0}}{G h_{0}}, \quad A=\frac{1}{h(x)} \int_{0}^{h(x)} \frac{\partial v}{\partial t} d y, \\
u=0, \quad v=-\frac{u^{*}(t)}{\tilde{u}^{0}}=e_{1}(t) \text { for } y=0 \\
u=0, \quad v=0 \text { for } y=h(x)=1+\eta x, \quad \eta=\frac{\operatorname{tg} \alpha}{h_{0}}, \\
\quad u=0, \quad v=0 \text { for } P=0 \text { for } t=0, \\
P=0 \text { for } x=0, x=1, \quad \frac{\partial^{2} P}{\partial x \partial t}+\operatorname{Re} \cdot \frac{\partial A}{\partial t}=0 \text { for } x=0, t=0 .
\end{gathered}
$$

An exact self-similar solution of problem (22), (23) will be sought in the following form:

$$
\begin{gathered}
v=\frac{\partial \psi}{\partial y}+V(x, y), \quad u=-\frac{\partial \psi}{\partial x}+U(x, y), \quad \psi=\widetilde{\psi}(\xi), \quad U=\widetilde{u}(\xi) h^{\prime}(x), \quad V=\widetilde{v}(\xi), \\
\xi=\frac{y}{h(x)}, \quad \frac{\partial P}{\partial x}+\operatorname{Re} \cdot A+\beta \frac{\partial^{2} P}{\partial x \partial t}+\operatorname{Re} \cdot \beta \cdot \frac{\partial A}{\partial t}=\frac{\widetilde{c}_{1}(t)}{h^{2}}+\frac{\widetilde{c}_{2}(t)}{h^{3}} .
\end{gathered}
$$

After substituting (24) into (22), (23), the following system of differential equations and the related boundary conditions are obtained:

$$
\begin{gathered}
\frac{\partial^{3} \widetilde{\psi}}{\partial \xi^{3}}=\widetilde{c}_{2}(t), \quad \frac{\partial^{2} \widetilde{v}}{\partial \xi^{2}}=\widetilde{c}_{1}(t), \quad \frac{\partial \widetilde{u}}{\partial \xi}-\xi \frac{\partial \widetilde{v}}{\partial \xi}=0 \\
\frac{\partial \widetilde{\psi}}{\partial \xi}=0, \quad \widetilde{u}=0, \widetilde{v}=0 \text { for } \xi=1, \quad \frac{\partial \widetilde{\psi}}{\partial \xi}=0, \widetilde{u}=0, \widetilde{v}=e_{1}(t) \text { for } \xi=0, \quad \int_{0}^{1} \widetilde{v}(\xi, t) d \xi=0 \\
P=0, \quad \widetilde{u}=0, \quad \widetilde{v}=0 \text { for } t=0 \\
P=0 \text { for } x=0, x=1, \quad \frac{\partial^{2} P}{\partial x \partial t}+\operatorname{Re} \frac{\partial A}{\partial t}=0 \text { for } x=0, t=0
\end{gathered}
$$

Integrating equation system (25) with boundary conditions (26), one obtains:

$$
\begin{gathered}
\widetilde{\psi}_{\xi}^{\prime}=\widetilde{c}_{2}(t) \frac{\xi^{2}}{2}+c_{1}(t) \xi+c_{2}(t), \quad \widetilde{v}(\xi, t)=\widetilde{c}_{1}(t) \frac{\xi^{2}}{2}+c_{3}(t) \xi+c_{4}(t), \\
\tilde{u}(\xi, t)=\int_{0}^{\xi} \frac{\partial \widetilde{v}(\xi, t)}{\partial \xi} d \xi,
\end{gathered}
$$

where

$$
\begin{gathered}
\widetilde{c}_{1}(t)=6 e_{1}(t), \quad c_{2}(t)=0, \quad c_{3}(t)=-4 e_{1}(t), \\
c_{1}(t)=-\frac{\widetilde{c}_{2}(t)}{2}, \quad c_{4}(t)=e_{1}(t), \quad A=-\frac{\widetilde{c}_{2}(t)}{12 h},
\end{gathered}
$$


and function $\widetilde{c}_{2}(t)$ is found from the following equation:

$$
\operatorname{Re} \cdot \beta \cdot \widetilde{c}_{2}^{\prime \prime}+\operatorname{Re} \cdot \widetilde{c}_{2}^{\prime}+\frac{12 J_{3}(1)}{J_{1}(1)} \widetilde{c}_{2}+72 e_{1} \frac{J_{2}(1)}{J_{1}(1)}=0 .
$$

After integrating equation (29), the following expression is obtained:

$$
\widetilde{c}_{2}=A_{1} \exp \left(b_{1} t\right)+A_{2} \exp \left(b_{2} t\right)-\frac{6 J_{2}(1) e_{1}}{J_{3}(1)},
$$

where

$$
\begin{aligned}
& b_{1}=\frac{-\operatorname{Re} \cdot J_{1}(1)+\sqrt{\operatorname{Re}^{2} \cdot J_{1}(1)^{2}-48 \operatorname{Re} \cdot \beta J_{3}(1) J_{1}(1)}}{2 \operatorname{Re} \cdot \beta}, \\
& b_{2}=\frac{-\operatorname{Re} \cdot J_{1}(1)-\sqrt{\operatorname{Re}^{2} \cdot J_{1}(1)^{2}-48 \operatorname{Re} \cdot \beta J_{3}(1) J_{1}(1)}}{2 \operatorname{Re} \cdot \beta} .
\end{aligned}
$$

For expression $\widetilde{c}_{2}(t)$ not to contain any complex roots, condition $A_{1}=A_{2}=0$ is enforced; then expression $\widetilde{c}_{2}(t)$ will take the form:

$$
\widetilde{c}_{2}=-\frac{6 J_{2}(1) e_{1}}{J_{3}(1)} .
$$

Hydrodynamic pressure $P$ is determined from the following equation:

$$
\frac{\partial P}{\partial x}+\beta \frac{\partial^{2} P}{\partial x \partial t}-\operatorname{Re} \cdot \frac{\tilde{c}_{2}^{\prime}}{12 h}-\operatorname{Re} \cdot \beta \frac{\tilde{c}_{2}^{\prime \prime}}{12 h}=\frac{6 e_{1}(t)}{h^{2}(x)}+\frac{\tilde{c}_{2}}{h^{3}(x)} .
$$

Integration of equation (31) for $x$, and then for $t$ with the account of boundary conditions (26) for $P$, yields:

$$
P=\frac{\exp (-t / \beta)}{\beta} \int_{0}^{t} E \exp (t / \beta) d t
$$

where

$$
E=\operatorname{Re} \cdot \beta \frac{J_{1}(x)}{12} \widetilde{c}_{2}^{\prime \prime}+\operatorname{Re} \cdot \frac{J_{1}(x)}{12} \widetilde{c}_{2}^{\prime}+J_{3}(x) \widetilde{c}_{2}+6 e_{1}(t) J_{2}(x) .
$$

Now, additional pressure forces $N$ acting on a unit width of the pilot and friction force $F_{\text {fr }}$ will be determined:

$$
\begin{gathered}
N=\int_{0}^{l} p_{\mathrm{ns}}^{\prime} d x^{\prime}=\frac{\widetilde{u}^{0} \mu l^{2}}{h_{0}^{2}} \int_{0}^{1} P d x=\frac{\widetilde{u}^{0} \mu l^{2}}{h_{0}^{2}} \times \\
\times\left[\operatorname { e x p } ( - t / \beta ) \int _ { 0 } ^ { t } \frac { \operatorname { e x p } ( t / \beta ) } { \beta } \left(\operatorname{Re} \cdot \beta \frac{\widetilde{c}_{2}^{\prime \prime}}{12} \int_{0}^{1} J_{1}(x) d x+\operatorname{Re} \cdot \frac{\widetilde{c}_{2}^{\prime}}{12} \int_{0}^{1} J_{1}(x) d x+\right.\right. \\
\left.\left.+\widetilde{c}_{2} \int_{0}^{1} J_{3}(x) d x+6 e_{1}(t) \int_{0}^{1} J_{2}(x) d x\right)\right], \\
F_{\mathrm{fr}}=\left.\int_{0}^{l} \tau_{\mathrm{ns}}^{\prime}\right|_{y^{\prime}=0} d x^{\prime}=\frac{\widetilde{u}^{0} \mu l}{h_{0}} \int_{0}^{1} c d x,
\end{gathered}
$$


where $c$ is found from the following equation:

$$
\frac{c_{1}}{h^{2}}+\frac{c_{3}}{h}=c+\beta \frac{\partial c}{\partial t} \text {. }
$$

After solving this equation with boundary condition $\partial c / \partial t=0$ for $x=0, t=0$, one obtains:

$$
c=\exp (-t / \beta) \int_{0}^{t} \frac{3 \exp (t / \beta) J_{2}(1) e_{1}(t)-4 \exp (t / \beta) h J_{3}(1) e_{1}(t)}{h^{2} J_{3}(1) \beta} \exp (t / \beta) d t .
$$

After substituting (36) into (34), the following final expression for determining the friction force is obtained:

$$
F_{\mathrm{fr}}=\frac{\widetilde{u}^{0} \mu l}{h_{0}} \exp (-t / \beta)\left[\left(\frac{3 J_{2}^{2}(1)}{J_{3}(1) \beta}-\frac{4 J_{1}(1)}{\beta}\right)_{0}^{t} \exp (t / \beta) e_{1} d t\right] .
$$

To solve the problem, it remains to find velocity $u^{*}=e_{1} \widetilde{u}^{0}$ of the bearing pilot. Let the mass of the pilot be equal to $M$, and the constant force acting on it be equal to $Q$. The following designations will be used:

$$
\frac{M}{l H}=m, \quad \frac{Q}{l H}=q
$$

where $H$ is width of the pilot.

The differential equation of motion of the plate in the designations used will have the form:

$$
\frac{d u^{*}}{d t^{\prime}}=\frac{q}{m}-\frac{1}{m l}\left(F_{\mathrm{fr}}+F_{\mathrm{fr}}^{0}\right) .
$$

In dimensionless variables, equation (39) with the account of (37) will be written as:

$$
\begin{gathered}
\frac{d e_{1}}{d t}=\frac{h_{0} q}{m\left(\widetilde{u}^{0}\right)^{2}}-\frac{\mu}{m \widetilde{u}^{0}}\left\{\exp (-t / \beta)\left[\left(\frac{3 J_{2}^{2}(1)}{J_{3}(1) \beta}-\frac{4 J_{1}(1)}{\beta}\right) \int_{0}^{t} \exp (t / \beta) e_{1} d t\right]+\right. \\
\left.+\left[-2 \frac{-4 \beta^{2} \eta-4 \eta \beta \exp (1 / \beta)+2 \eta \exp (1 / \beta)+4 \eta \beta^{2} \exp (1 / \beta)-\exp (1 / \beta)}{-6 \beta^{2} \eta+3 \eta \exp (1 / \beta)-2 \exp (1 / \beta)-6 \eta \beta \exp (1 / \beta)+6 \eta \beta^{2} \exp (1 / \beta)}\right]\right\} .
\end{gathered}
$$

Differentiation of both sides of equation (40) for $t$ yields:

$$
\frac{d^{2} e_{1}}{d t^{2}}=-\frac{\mu}{m \widetilde{u}^{0}}\left\{\left(\frac{3 J_{2}^{2}(1)}{J_{3}(1) \beta}-\frac{4 J_{1}(1)}{\beta}\right)\left(-\frac{1}{\beta} \exp (-t / \beta) \int_{0}^{t} \exp (t / \beta) e_{1} d t+e_{1}\right)\right\} .
$$

Excluding integral $\exp (-t / \beta) \int_{0}^{t} e_{1} \exp (t / \beta) d t$ from (40) and (41), the final form of the equation is obtained:

$$
\begin{gathered}
\frac{d^{2} e_{1}}{d t^{2}}+\frac{1}{\beta} \frac{d e_{1}}{d t}+\frac{\mu}{m \widetilde{u}^{0}} e_{1}\left[\frac{3 J_{2}^{2}(1)}{J_{3}(1) \beta}-\frac{4 J_{1}(1)}{\beta}\right]=\frac{h_{0} q}{\beta m\left(\widetilde{u}^{0}\right)^{2}}-\frac{\mu}{\beta m \widetilde{u}^{0}} \times \\
\times\left[-2 \frac{-4 \beta^{2} \eta-4 \eta \beta \exp (1 / \beta)+2 \eta \exp (1 / \beta)+4 \eta \beta^{2} \exp (1 / \beta)-\exp (1 / \beta)}{-6 \beta^{2} \eta+3 \eta \exp (1 / \beta)-2 \exp (1 / \beta)-6 \eta \beta \exp (1 / \beta)+6 \eta \beta^{2} \exp (1 / \beta)}\right] .
\end{gathered}
$$

Solution of equation (42) yields: 


$$
\begin{gathered}
e_{1}=\frac{h_{0} q}{\beta \delta m\left(\widetilde{u}^{0}\right)^{2}}-\frac{\mu}{\beta \delta m \widetilde{u}^{0}} \times \\
\times\left[-2 \frac{-4 \beta^{2} \eta-4 \eta \beta \exp (1 / \beta)+2 \eta \exp (1 / \beta)+4 \eta \beta^{2} \exp (1 / \beta)-\exp (1 / \beta)}{-6 \beta^{2} \eta+3 \eta \exp (1 / \beta)-2 \exp (1 / \beta)-6 \eta \beta \exp (1 / \beta)+6 \eta \beta^{2} \exp (1 / \beta)}\right]+ \\
+E_{1} \exp \left(a_{1} t\right)+E_{2} \exp \left(a_{2} t\right)
\end{gathered}
$$

where

$$
\delta=\frac{\mu}{\beta m \widetilde{u}^{0}}\left(\frac{3 J_{2}^{2}(1)}{J_{3}(1)}-4 J_{1}(1)\right), \quad a_{1}=-\frac{1}{2} \cdot \frac{1+\sqrt{1-4 \delta \beta^{2}}}{\beta}, \quad a_{2}=\frac{1}{2} \cdot \frac{-1+\sqrt{1-4 \delta \beta^{2}}}{\beta} .
$$

As in the considered case, for actual bearings, acquires values from 0 to 1 , then, for these values, $\delta<0$, hence, $a_{1}<0, a_{2}>0$, and, thus, in the solution of (43) it is assumed that $E_{2}=0$. Using boundary conditions $e_{1}=0$ for $t=0$, one obtains:

$$
\begin{gathered}
E_{1}=-\frac{h_{0} q}{\beta \delta m\left(\widetilde{u}^{0}\right)^{2}}+\frac{\mu}{\beta \delta m \tilde{u}^{0}} \times \\
\times\left[-2 \frac{-4 \beta^{2} \eta-4 \eta \beta \exp (1 / \beta)+2 \eta \exp (1 / \beta)+4 \eta \beta^{2} \exp (1 / \beta)-\exp (1 / \beta)}{-6 \beta^{2} \eta+3 \eta \exp (1 / \beta)-2 \exp (1 / \beta)-6 \eta \beta \exp (1 / \beta)+6 \beta^{2} \eta \exp (1 / \beta)}\right] .
\end{gathered}
$$

Then, the final expression for $e_{1}$ will be:

$$
\begin{gathered}
e_{1}=\left[1-\exp \left(a_{1} t\right)\right]\left(-\frac{h_{0} q}{\beta \delta m\left(\tilde{u}^{0}\right)^{2}}+\frac{\mu}{\beta \delta m \tilde{u}^{0}} \times\right. \\
\left.\times\left[-2 \frac{-4 \beta^{2} \eta-4 \eta \beta \exp (1 / \beta)+2 \eta \exp (1 / \beta)+4 \eta \beta^{2} \exp (1 / \beta)-\exp (1 / \beta)}{-6 \beta^{2} \eta+3 \eta \exp (1 / \beta)-2 \exp (1 / \beta)-6 \eta \beta \exp (1 / \beta)+6 \beta^{2} \eta \exp (1 / \beta)}\right]\right) .
\end{gathered}
$$

The total pressure force and the total friction are found from the following expressions:

$$
W=N+N_{0}, \quad F=F_{\text {fr }}+F_{\text {fr }}^{0} .
$$

\section{Analysis of the results obtained}

Some of the obtained results will further be analyzed here. Firstly, it follows from (44) that, for $t \rightarrow \infty$, the velocity of the pilot tends to limiting value $U$, which is equal to:

$$
\begin{gathered}
U=-\frac{h_{0} q}{\beta \delta m\left(\widetilde{u}^{0}\right)^{2}}+\frac{\mu}{\beta \delta m \widetilde{u}^{0}} \times \\
\times\left[-2 \frac{-4 \beta^{2} \eta-4 \eta \beta \exp (1 / \beta)+2 \eta \exp (1 / \beta)+4 \eta \beta^{2} \exp (1 / \beta)-\exp (1 / \beta)}{-6 \beta^{2} \eta+3 \eta \exp (1 / \beta)-2 \exp (1 / \beta)-6 \eta \beta \exp (1 / \beta)+6 \beta^{2} \eta \exp (1 / \beta)}\right] .
\end{gathered}
$$

The results of numerically analyzing total pressure force as a function of Deborah number $\beta$ and time $t$ are depicted in Figs. 2, 3. The total pressure force acting on the slider and the pilot at the moment when the pilot moves at this velocity $\widetilde{u}^{0}+u^{*}\left(t^{\prime}\right)$ is higher than the force that would be acting on it if the pilot moved steadily, beginning from this moment, at a constant velocity of $\widetilde{u}^{0}$. It is evident from expressions (45), with the account of (44) defining the pressure and friction forces, that in the limit $(t \rightarrow \infty)$ those expressions transform into formulas defining the pressure and friction forces in the case of stationary motion, which testifies to a steady-state working regime of the system in question. 


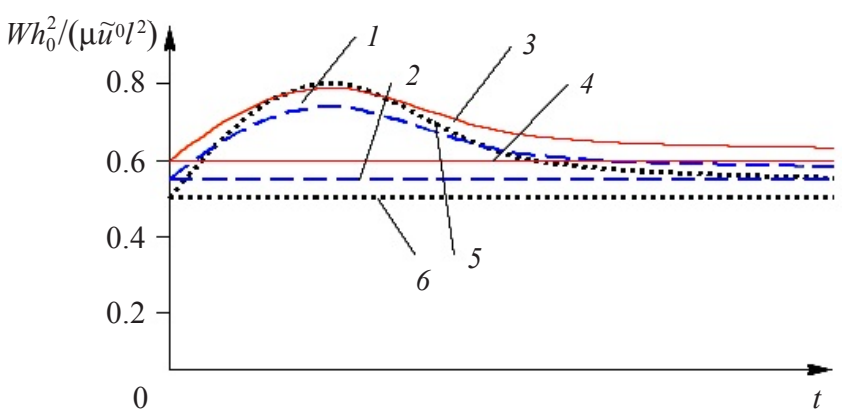

Fig. 2. Total pressure force as a function of time for various Deborah numbers:

$1-\beta=0.5$ - nonstationary working regime, $2-\beta=0.5-$ steady-state working regime,

$3-\beta=0.8$ - nonstationary working regime, $4-\beta=0.8$ - steady-state working regime,

$5-\beta=0.2-$ nonstationary working regime, $6-\beta=0.2-$ steady-state working regime

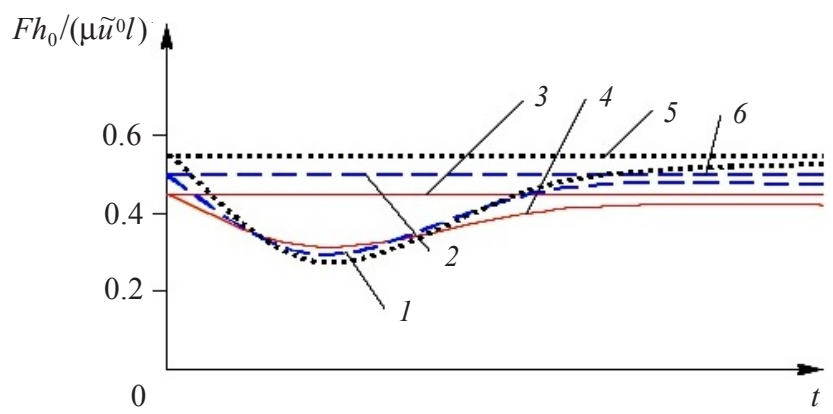

Fig. 3. Total friction force as a function of time for various Deborah numbers:

$1-\beta=0.5$ - nonstationary working regime, $2-\beta=0.5-$ steady-state working regime,

$3-\beta=0.8-$ steady-state working regime, $4-\beta=0.8$ - nonstationary working regime, $5-\beta=0.2-$ steady-state working regime, $6-\beta=0.2-$ nonstationary working regime

\section{Conclusion}

The above analysis of the effect of viscoelastic properties of lubricants in the framework of the Maxwell model has shown that this effect is the most pronounced at the initial stage of loading when nonstationary effects play the key role.

\section{References}

1. Nikitin A.K., Akhverdiev K.S., Ostroumov B.I. Gidrodinamicheskaya teoriya smazki i raschet podshipnikov skolzheniya, rabotayushchikh $v$ statsionarnom rezhime [Hydrodynamic Theory of Lubrication and Calculation of Sliding Bearings Operating in Stationary Mode]. Moscow. Nauka Publ. 1981. 316 p. (In Russian).

2. Akhverdiev, K.S., Zhurba I.A., Yakovlev M.V. Gidrodinamicheskiy raschet podshipnikov skolzheniya s uchetom sil inertsii smazochnoy zhidkosti, obladayushchey vyazkouprugimi svoystvami [Hydrodynamic calculation of sliding bearings taking into account the forces of inertia of the lubricating fluid having viscoelastic properties]. Trenie $i$ iznos [Journal of Friction and Wear]. 2003. Vol. 24. No 2. P. 121-125 (In Russian).

3. Tichy J.A., Winer W.O. An investigation into the influence of fluid viscoelasticity in a squeeze film bearing. Journal of Lubrication Technology. 1978. Vol. 100. Iss. 1. P. 56-64.

4. Tao F.F., Philippoff W. Hydrodynamic behavior of viscoelastic liquids in a simulated journal bearing. ASLE Transactions. 1967. Vol. 10. Iss. 3. P. 302-315.

5. Cal F.S., Dias G.A., Pereira B.M., Pires G.E., Rajagopal K.R., Videman J.H. On the 
lubrication approximation for a class of viscoelastic fluids. International Journal of Non-Linear Mechanics. 2016. Vol. 87. P. 30-37.

6. Habchi W., Bair S. Is viscoelasticity of any relevance to quantitative EHL friction predictions? Tribology International. 2019. Vol. 135. P. 96-103.

7. Tanner R.I. Full-film lubrication theory for a Maxwell liquid. International Journal of Mechanical Sciences. 1960. Vol. 1. Iss. 2-3. P. 206-215.

8. Mitchell D. Rolling contact lubrication using a Maxwell fluid. Journal of Mechanical Engineering Science. 1970. Vol. 12. Iss. 4. P. 235-237.

9. Seabra J., Sottomayor A., Campos A. Non-Newtonian EHL model for traction evaluation in a roller-inner ring contact in a roller bearing. Wear. 1996. Vol. 195. Iss. 1-2. P. 53-65.

10. Makris N., Constantinou M.C. Fractional-derivative Maxwell model for viscous dampers. Journal of Structural Engineering. 1991. Vol. 117. No 9. P. 2708-2724.

11. Lamb J. Paper 18: Physical properties of fluid lubricants: rheological and viscoelastic behaviour. Proceedings of the Institution of Mechanical Engineers. Conference Proceedings. 1967. Vol. 182. No 1. London. SAGE Publications. P. 293-310.

12. Lin J.R. Non-Newtonian effects on the dynamic characteristics of one-dimensional slider bearings: Rabinowitsch fluid model. Tribology Letters. 2001. Vol. 10. Iss. 4. P. 237-243.

13. Safar Z.S., Shawki G.S.A. Performance of thrust bearings operating with non-Newtonian lubricating films. Tribology International. 1979. Vol. 12. No 1. P. 31-33.

14. Swamy S.T.N., Prabhu B.S., Rao B.V.A. Calculated load capacity of non-Newtonian lubricants in finite width journal bearings. Wear. 1975. Vol. 31. Iss. 2. P. 277-285.

15. Sinhasan R., Sah P.L. Static and dynamic performance characteristics of an orifice compensated hydrostatic journal bearing with non-Newtonian lubricants. Tribology International. 1996. Vol. 29. No 6. P. 515-526.

16. Astarita G., Marucci G. Principles of Non-Newtonian Fluid Mechanics. New York. McGraw-Hill. 1974. 289 p.

17. Wilkinson W.L. Non-Newtonian Fluids. London. Pergamon Press. 1960. 138 p.

18. Truesdell C. A First Course in Rational Continuum Mechanics. New York. Academic Press. 1977. 304 p.

19. Joseph D.D., Liao T.Y. Potential flows of viscous and viscoelastic fluids. Journal of Fluid Mechanics. 1994. Vol. 265. P. 1-23.

20. Akhverdiev K.S., Zhurba I.A. Ustanovivsheesya dvizhenie vyazkouprugoy zhidkosti mezhdu naklonnym polzunom i napravlyayushchey, soglasovannoe s silami inertsii smazki [Steadystate motion of a viscoelastic liquid between a tilt slider and a guide consistent with the lubricant inertia forces]. Trenie i iznos [Journal of Friction and Wear]. 2004. Vol. 25. No 6. P. 567-576 (In Russian).

\section{МАТЕМАТИЧЕСКАЯ МОДЕЛЬ НЕСТАЦИОНАРНОГО ДВИЖЕНИЯ ВЯЗКОУПРУГОЙ ЖИДКОСТИ В ПОДШИПНИКАХ СКОЛЬЖЕНИЯ}

\section{Журба Еремеева И.А., Скеррато Д., Кардилло К., Тран А.}

Международный исследовательский центр математики и механики сложных систем, университет Л' Акуила, Л' Акуила, Италия

В настоящее время появление новых смазочных материалов требует расширения используемых реологических моделей и методов решения соответствующих начальнокраевых задач. В частности, большой интерес представляют модели, учитывающие вязкоупругие свойства. В статье рассматривается модель нестационарного движения вязкоупругой жидкости в подшипниках скольжения. Для описания свойств жидкости использована модель Максвелла. Вязкоупругие свойства проявляются во многих смазочных материалах, содержащих полимерные добавки. Кроме того, вязкоупругие свойства могут быть существенными при высоких скоростях жидкости. Также вязкоупругие свойства могут проявляться в случае тонких зазоров. Модель Максвелла представляет собой одну из наиболее 
распространенных моделей вязкоупругих материалов. Она сочетает относительную простоту уравнений состояния с возможностью описания релаксации напряжений. Помимо этого, уравнения вязкоупругой жидкости позволяют описать эффекты, отсутствующие в случае вязкой жидкости, например, эффект Вайссенберга и целый ряд других. В частности, такие эффекты можно использовать для повышения эффективности несущей пленки в подшипниках скольжения. В статье введены предположения о характере течения, позволяющие существенно упростить решение задачи. Рассматриваются автомодельные решения, что позволяет получить решение в аналитической форме. Получены зависимости полной силы давления и полной силы трения от числа Деборы и времени. Получены предельные значения ряда характеристик течения, которые можно использовать в установившемся режиме течения. Обсуждаются отличия от случая ньютоновской жидкости. Показано, что вязкоупругие свойства в большей мере проявляются на начальном этапе нагружения, когда важны эффекты нестационарности.

Ключевые слова: вязкоупругая жидкость, подшипник скольжения, теория смазки, автомодельное решение. 